Mineralogical Journal, Vol. 5, No. 2, PP. 98-123, Feb., 1967

\title{
THE STRUCTURE OF HARADAITE AND A NOTE ON THE Si-O BOND LENGTHS IN SILICATES
}

\author{
YoSHIO TAKÉUCHI and WERNER JOSWIG* \\ Mineralogical Institute, Faculty of Science, University \\ of Tokyo, Hongo, Tokyo
}

\begin{abstract}
Haradaite, $\mathrm{SrVSi}_{2} \mathrm{O}_{7}$, is orthorhombic, space group Amam, with four formula units in the cell, $a=7: 06, b=14.64, c=5.33 \AA$. The structure contains $\mathrm{Si}_{4} \mathrm{O}_{12}$ chains which are joined together by vanadium to form a sheet closely related to those in lamprophyllite, bafertisite and astrophyllite. These minerals may conveniently be called quasi-layer silicates.

There are two kinds of bridge bonds in the chain: one is a nonlinear bond $\left(\angle \mathrm{Si}-\mathrm{O}-\mathrm{Si} 150^{\circ}\right)$ of length $1.664 \pm 0.008 \AA$, the other is a linear bond of length $1.578 \pm 0.009 \AA$. The non-bridge bonds have a length $1.59_{8} \pm 0.03 \AA$. A general survey on the $\mathrm{Si}-\mathrm{O}$ bond lengths in silicates shows that, for nonlinear cases, the mean value of bridge bonds is $1.641 \AA$ and that of non-bridge bonds $1.605 \AA$, and for linear cases, these values are respectively $1.59 \AA$ and $1.63 \AA$.
\end{abstract}

\section{Introduction}

As part of our program of the study on layer silicates, we have analyzed the crystal structure of haradaite which is a new mineral found by WATANABE and KATO in 1960 at the Nodatamagawa manganese mine, Iwate, Japan. Although a full description of the mineral will be given elsewhere by WATANABE and others, the specimen and the necessary information were supplied for our structural investigation. It shows bright green color and has one perfect cleavage, giving a strong resemblance to the mica group. The material has the theoretical composition $\mathrm{SrVSi}_{2} \mathrm{O}_{7}$ in which $\mathrm{Sr}$ is replaced in a

* Present address: Mineralogisches Institut der Universität Würzburg, Pleichertorstr. 34, Würzburg, Germany. 
small amount by $\mathrm{Ba}$. The synthetic study of the mineral by ITO (1965) confirmed this chemical formula.

The structure of haradaite contains a sheet which is closely related to that in lamprophyllite (WOODRow, 1964), astrophyllite (WoODRow, 1963), and bafertisite (YA-HsIEN et al., 1963) in the respect that the sheets consist of Si-O tetrahedra and $\mathrm{Ti}$ (or V)-O polyhedra. The minerals characterized by this kind of sheet structure may be called 'quasi-layer silicates'. The relations between mica structure and quasi-layer structure are discussed. In connection with the Si-O bond lengths found in haradaite, a general survey on the bond lengths in silicates was made separately for Si-O-Si bridge bonds, and for non-bridge bonds, and a significant difference has been concluded between these two types of bonds.

\section{Unit cell and space group}

Haradaite is orthorhombic. The unit cell dimensions are:

$$
a=7.06 \AA, b=14.64 \AA, c=5.33 \AA \text {. }
$$

The cell is $A$-centred and contains four formula units, the calculated value $Z=4.1$ being obtained from the observed density $D=3.80 \mathrm{~g} / \mathrm{cm}^{3}$. On the basis of this set of axes, the perfect cleavage has the indices (010).

Rules of missing reflections suggest Amam-No. 63 or Ama2-No. 40 as the most probable space group. Piezo tests were made on the cleavage fragments. The effect was negative along each of the principal crystallographic axes. The structure analysis was therefore initiated by assuming the centrosymmetric space group. The possibilities of non-centrosymmetric space group, Ama2, were tested at the final stage of the refinements. The final conclusion is however, that the space group is the centrosymmetric one, Amam. 


\section{Experimental}

Intensity data were produced with an integrating Weissenberg camera, $\mathrm{Cu} K \alpha$ radiation being used. Attempts to cut cleavage flakes to size were unsuccessful, the flakes only splitting along the cleavage plane and bending. Even fairly good specimens which gave good reflections for $h k 0, h k 1$ and $h k 2$ failed in giving satisfactory reflections for higher levels such as $h k 3$ and $h k 4$. Therefore, for the structure determination and refinements, only the reflections $0 k l, h k 1$ and $h k 2$ were used. However, as the $c$-axis has a short repeat $(5.33 \AA)$, it was considered that these data would be sufficient to determine the essential feature of the structure. The dimensions of the specimen used for intensity measurements were approximately $0.25 \times 0.25$ $\times 0.05 \mathrm{~mm}^{3}$. Intensities were measured by the use of a microdensitometer and a computer program was employed to correct for the transmission factor.

\section{Structure determination}

\section{(1) $x y$-projection}

Since there are four $\mathrm{Sr}$ and $\mathrm{V}$ in the cell, they should be located either on the two-fold axis $(1 / 4, y, 0)$ or at the center of symmetry. In the $x y$-projection of the plane group pmg, these special positions of the space group Amam correspond to mirror planes and two-fold axes respectively. In this projection, therefore, there are four possible combinations for $\mathrm{Sr}$ and $\mathrm{V}$ locations:

$\begin{array}{ccc} & \mathrm{Sr} & \mathrm{V} \\ \text { (a) } & m & 2 \\ \text { (b) } & 2 & m \\ \text { (c) } & 2 & 2 \\ \text { (d) } & m & m\end{array}$

where $m$ and 2 indicate the mirror plane and two-fold axis of pmg respectively. 


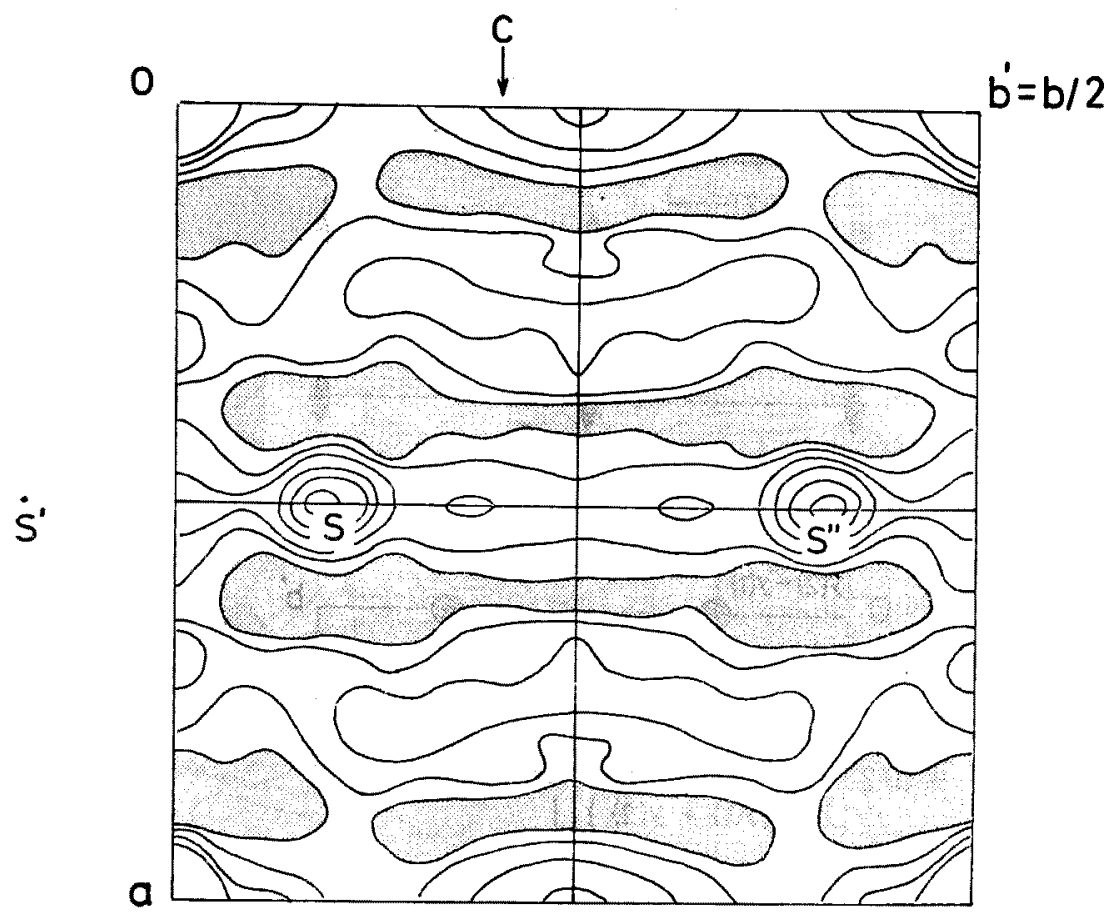

Fig. 1. Patterson projection $P(u v)$ of haradaite.

Among these combinations, it was found that (b) and (c) are not conformable to the Patterson diagram $P(u v)$ (Fig. 1) because both require a peak at $(1 / 2,0)$ higher than that observed. The combination (a) was then tested. The variation of the $R$-factor with that of the $y$-parameters of $\mathrm{Sr}$ was derived where for calculations only $\mathrm{Sr}$ and $\mathrm{V}$ were taken into account. It was then found that the $R$ value was reduced as low as 0.30 for $y=0.20$, but any reasonable structure could not be deduced from this combination. It was therefore to be expected that the correct structure of haradaite should be derived from the combination (b). For this particular case where two independent atoms lie on a mirror plane, the cross vectors between two atoms in the Patterson projection bear geometrical relations as shown in Fig. 2. It is often worthwhile to use the cross 

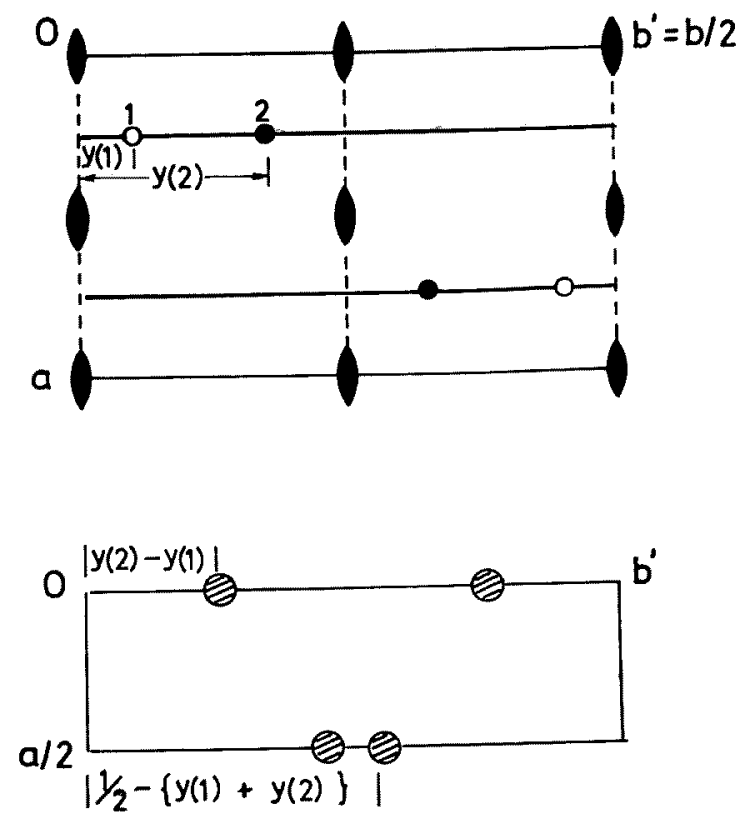

Fig. 2. Plane group $p m g$ indicating atoms 1 and 2 on the mirror planes (upper figure). Geometrical relation of cross-vector peaks between atom 1 and atom 2, in the Patterson space, is given (lower figure).

vector relations (TAKÉUCHI et al., 1965). Because of high multiplicity of the cross vector peaks, it is easy to identify them. For the present case, the multiplicity of the $\mathrm{Sr}-\mathrm{V}$ vectors is four. It was. found that the peaks $C(u=0, v=0.20), S(u=1 / 4, v=0.10)$ and their symmetrical pairs are the only possible candidates for such peaks. These peaks can be used to solve the $y$-parameters of these two. atoms.

Once we choose the peak $C$, we have to consider all symmetrical pairs of the peak $S$. Among them the combination of $C$ and $S^{\prime}$ at. $u=1 / 4, v=0.10$ gives the equations:

$$
\begin{aligned}
& y(2)-y(1)=0.20 \\
& y(2)+y(1)=1 / 2+0.1,
\end{aligned}
$$


and we obtain $y(1)=0.20, y(2)=0.40$. As expected from the result obtained for the case of (a), the assignment, $y(1)$ to Sr and $y(2)$ to $\mathrm{V}$, gives the best agreement between $F_{o}$ and $F_{c}$. For this case the

(a)

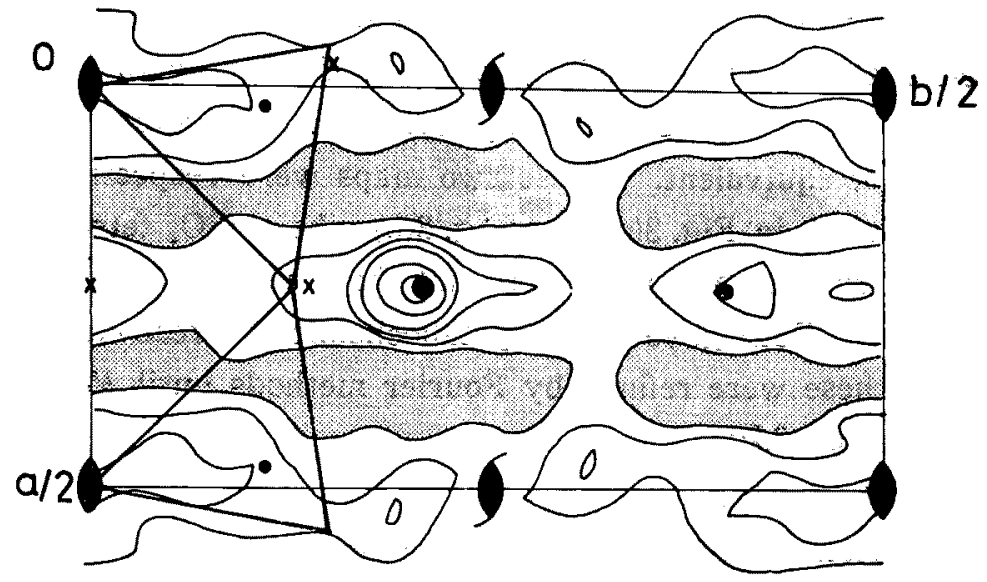

(b)

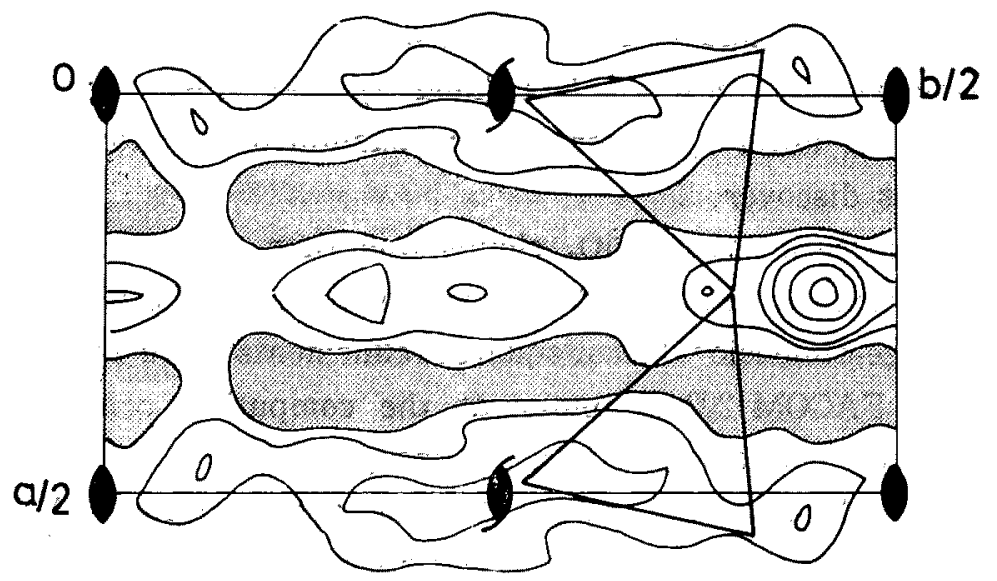

Fig. 3. Maps of minimum function $M_{2}(x y)$. Possible arrangements of silicon tetrahedra are indicated. The interpretation (b) turned out to be incorrect. Final atomic locations are shown: large, medium and small solid circles are respectively $\mathrm{Sr}, \mathrm{V}$ and $\mathrm{Si}$, crosses indicate oxygen. 
Sr-Sr symmetrical vector peaks are located at the peak $S$. Therefore, based upon this peak, the minimum functions $M_{2}(x y)$ were formed. They are shown in Fig. 3(a) and Fig. 3(b). In Fig. 3(b), the origin is shifted to $(0,1 / 4)$ from that of Fig. 3(a). As far as the plane group pmg is concerned, these two maps should be equivalent. However, the $b$-translation of the plane group of haradaite is merely a pseudo-translation corresponding to one half of the actual $b$-translation. For the actual space-group symmetry, therefore, these two maps are not equivalent. These two maps were respectively interpreted as shown in Fig. 3(a) and Fig. 3(b) where $\mathrm{SiO}_{4}$ tetrahedra are indicated. One gives $\mathrm{Si}_{2} \mathrm{O}_{7}$ groups and the other $\mathrm{SiO}_{3}$ chains parallel to the $a$-axis. Both gave the crystallochemically reasonable structures, and these were refined by Fourier methods until $R$-factor fell down to around 0.20 .

\section{(2) $y z$-projection}

The Patterson projection $P(v w)$ suggests that the majority of atoms are located on the levels $z=0$ (or 0.5) and $z=0.25$ (or 0.75). The structure factors of the structure having $\mathrm{Si}_{2} \mathrm{O}_{7}$ groups as described above were first calculated. The results gave a good agreement between $F_{o}$ and $F_{c}$ for the reflections $l=0$ ( $\left.\bmod 2\right)$, but very unsatisfactory for those of $l=1$. (mod 2). At this stage, various possibilities of lower symmetries were examined but no successful solution was discovered.

The structure having $\mathrm{SiO}_{3}$ chains was then tested, and a satisfactory agreement between $F_{o}$ and $F_{c}$ was produced for all $0 k l$ reflections. Three-dimensional least squares refinements were then carried out on the FACOM 202 computer at the computing center of the University of Tokyo with the aid of the program written by Iitaka. In this calculation, for atomic scattering factors, Forsyth-Wells approximation was used with parameters given by HosoYA and SATAKE (1965). The final atomic parameters gave $R=8.6 \%$ if unobserved reflections were neglected, and $9.4 \%$ for all reflections. The final atomic parameters are given in Table 1 and the comparison of $F_{\circ}$ 's 
Table 1. Atomic parameters of haradaite

\begin{tabular}{|c|c|c|c|c|}
\hline Atom & $x$ & $y$ & $z$ & $B$ \\
\hline $\mathrm{Sr}$ & 0.25 & $\begin{array}{r}0.2058 \\
\pm 0.0001\end{array}$ & 0 & $\begin{array}{r}1.27 \\
\pm 0.05\end{array}$ \\
\hline $\mathrm{V}$ & 0.25 & $\begin{array}{r}0.3931 \\
\pm 0.0003\end{array}$ & 0.5 & $\begin{array}{r}1.35 \\
\pm 0.12\end{array}$ \\
\hline $\mathrm{Si}$ & $\begin{array}{r}0.022_{3} \\
\pm 0.0011\end{array}$ & $\begin{array}{r}0.1073 \\
\pm 0.0004\end{array}$ & 0.5 & $\begin{array}{r}1.41 \\
\pm 0.14\end{array}$ \\
\hline $\mathrm{O}_{1}$ & $\begin{array}{l}-0.058_{7} \\
\pm 0.0019\end{array}$ & $\begin{array}{r}0.1511 \\
\pm 0.0006\end{array}$ & $\begin{array}{r}0.252_{8} \\
\pm 0.0039\end{array}$ & $\begin{array}{r}1.36 \\
\pm 0.27\end{array}$ \\
\hline $\mathrm{O}_{2}$ & 0 & 0 & 0.5 & $\begin{aligned} & 0.5 \\
\pm & 0.00\end{aligned}$ \\
\hline $\mathrm{O}_{3}$ & 0.25 & $\begin{array}{l}-0.0003 \\
\pm 0.0015\end{array}$ & 0 & $\begin{aligned} & 0.5 \\
\pm & 0.00\end{aligned}$ \\
\hline $\mathrm{O}_{4}$ & 0.25 & $\begin{array}{r}0.1366 \\
\pm 0.0014\end{array}$ & 0.5 & $\begin{aligned} & 0.5 \\
\pm & 0.00\end{aligned}$ \\
\hline
\end{tabular}

and $F_{c}$ 's in Table 2.

(3) Test for the lower symmetry, Ama2, and discussions.

In the structure thus determined, the oxygen $\mathrm{O}_{2}$ has a bond angle of $180^{\circ}$ simply because it is located on the center of symmetry. Since this type of oxygen bond angle is very rare in silicates, an attempt was made to extend the least squares refinement to the space group Ama2 using a modified version of Busing-Martin-Levy program. Starting from the atomic parameters obtained for the space group Amam, five cycles of least squares calculations were made among which the third one gave the smallest $R$ value. The resulting parameters are given in Table 3. The weighting scheme used was: $\sqrt{w}=F_{o} / F_{o \min }$ if $F_{o} \leqq F_{o \min }, \sqrt{w}=F_{o \min } / F_{o}$ if $F_{o}>F_{o \text { min }}$ and $\sqrt{w}=F_{o \min } \cdot F_{o \max } / F_{o}^{2}$ if $F_{o}>F_{o \max }$. In addition to that, several reflections which show relatively poor agreement between $F_{o}$ and $F_{c}$. were weighted zero. It was observed that the fairly strong inter- 
Table 2. Comparison of $\left|F_{o}\right|$ and $F_{c}$ values of haradaite.

\begin{tabular}{|c|c|c|c|c|c|c|c|c|c|}
\hline$h$ & $k$ & $l$ & $F_{o}$ & $F_{c}$ & $h$ & $k$ & $l$ & $F_{o}$ & $F_{c}$ \\
\hline 0 & 2 & 0 & 69.74 & -66.79 & 0 & 0 & 4 & 295.54 & 247.74 \\
\hline 0 & 4 & 0 & 149.57 & -144.74 & 0 & 2 & 4 & 45.84 & -35.90 \\
\hline 0 & 6 & 0 & 54.75 & 64.00 & 0 & 4 & 4 & 69.75 & -66.78 \\
\hline 0 & 8 & 0 & 80.67 & 85.72 & 0 & 6 & 4 & 0 & -2.21 \\
\hline 0 & 10 & 0 & 130.19 & 134.43 & 0 & 8 & 4 & 41.94 & 43.64 \\
\hline 0 & 12 & 0 & 60.26 & -63.69 & 0 & 10 & 4 & 87.26 & 92.98 \\
\hline 0 & 14 & 0 & 18.70 & 17.97 & 0 & 12 & 4 & 45.87 & -44.76 \\
\hline 0 & 16 & 0 & 19.28 & -23.46 & 0 & 1 & 5 & 0 & -9.07 \\
\hline 0 & 18 & 0 & 30.90 & 34.46 & 0 & 3 & 5 & 37.78 & -36.03 \\
\hline 0 & 1 & 1 & 22.30 & 20.15 & 0 & 5 & 5 & 74.18 & 65.01 \\
\hline 0 & 3 & 1 & 71.34 & -73.71 & 0 & 7 & 5 & 45.57 & -51.67 \\
\hline 0 & 5 & 1 & 123.40 & 127.66 & 0 & 0 & 6 & 102.97 & 101.06 \\
\hline 0 & 7 & 1 & 97.82 & -106.62 & 0 & 2 & 6 & 0 & -8.75 \\
\hline 0 & 9 & 1 & 40.65 & 40.67 & 0 & 4 & 6 & 0 & -6.31 \\
\hline 0 & 11 & 1 & 0 & -19.97 & 0 & 6 & 6 & 30.19 & -25.35 \\
\hline 0 & 13 & 1 & 21.94 & -25.51 & 0 & 8 & 6 & 0 & -3.56 \\
\hline 0 & 15 & 1 & 35.72 & 45.09 & 2 & 0 & 0 & 67.38 & -73.53 \\
\hline 0 & 17 & 1 & 36.08 & -40.33 & 4 & 0 & 0 & 248.25 & 256.74 \\
\hline 0 & 0 & 2 & 234.75 & 232.20 & 6 & 0 & 0 & 118.85 & -105.03 \\
\hline 0 & 2 & 2 & 0 & -5.36 & 8 & 0 & 0 & 88.82 & 83.53 \\
\hline 0 & 4 & 2 & 14.21 & 16.81 & 2 & 2 & 0 & 89.22 & 83.44 \\
\hline 0 & 6 & 2 & 71.17 & -72.69 & 3 & 2 & 0 & 58.14 & 62.17 \\
\hline 0 & 8 & 2 & 32.15 & 43.04 & 4 & 2 & 0 & 30.54 & -27.75 \\
\hline 0 & 10 & 2 & 187.89 & 202.77 & 5 & 2 & 0 & 0 & -3.21 \\
\hline 0 & 12 & 2 & 85.00 & -82.71 & 6 & 2 & 0 & 74.67 & 63.61 \\
\hline 0 & 14 & 2 & 20.92 & -20.74 & 1 & 4 & 0 & 120.66 & 119.95 \\
\hline 0 & 18 & 2 & 39.92 & 37.59 & 2 & 4 & 0 & 86.60 & -83.46 \\
\hline 0 & 1 & 3 & 0 & -1.88 & 3 & 4 & 0 & 177.54 & -170.45 \\
\hline 0 & 3 & 3 & 59.74 & -48.04 & 4 & 4 & 0 & 41.03 & -39.16 \\
\hline 0 & 5 & 3 & 102.96 & 98.57 & 5 & 4 & 0 & 47.63 & -46.36 \\
\hline 0 & 7 & 3 & 75.12 & -83.36 & 6 & 4 & 0 & 0 & 1.83 \\
\hline 0 & 9 & 3 & 36.41 & -35.37 & 7 & 4 & 0 & 91.35 & 48.92 \\
\hline 0 & 11 & 3 & 0 & 0.03 & 1 & 6 & 0 & 153.41 & -148.62 \\
\hline 0 & 13 & 3 & 20.40 & -22.84 & 2 & 6 & 0 & 16.42 & 22.14 \\
\hline 0 & 15 & 3 & 32.93 & 37.43 & 3 & 6 & 0 & 114.53 & 107.48 \\
\hline
\end{tabular}


Table 2. Continued

\begin{tabular}{|c|c|c|c|c|c|c|c|c|c|}
\hline$h$ & $k$ & $l$ & $F_{o}$ & $F_{c}$ & $h$ & $k$ & $l$ & $F_{o}$ & $F_{c}$ \\
\hline 4 & 6 & 0 & 0 & -10.71 & 8 & 1 & 1 & 21.45 & 16.42 \\
\hline 5 & 6 & 0 & 91.66 & -87.38 & 1 & 3 & 1 & 173.10 & 173.10 \\
\hline 6 & 6 & 0 & 20.85 & -21.77 & 2 & 3 & 1 & 86.26 & 73,16 \\
\hline 7 & 6 & 0 & 73.61 & 79.76 & 3 & 3 & 1 & 114.00 & -104.46 \\
\hline 1 & 8 & 0 & 61.16 & 55.82 & 4 & 3 & 1 & 57.43 & -53.34 \\
\hline 2 & 8 & 0 & 45.92 & 53.46 & 5 & 3 & 1 & 123.71 & 123.11 \\
\hline 3 & 8 & 0 & 31.63 & 34.59 & 6 & 3 & 1 & 60.25 & 48.96 \\
\hline 4 & 8 & 0 & 39.75 & 41.84 & 7 & 3 & 1 & 41.60 & -39.04 \\
\hline 5 & 8 & 0 & 76.20 & 71.08 & 8 & 3 & 1 & 41.17 & $-32,32$ \\
\hline 1 & 10 & 0 & 20.10 & -19.61 & 1 & 5 & 1 & 60.46 & -61.36 \\
\hline 2 & 10 & 0 & 80.23 & -88.21 & 2 & 5 & 1 & 30.34 & -32.02 \\
\hline 3 & 10 & 0 & 0 & 1. 82 & 3 & 5 & 1 & 39.01 & 39.31 \\
\hline 4 & 10 & 0 & 129.06 & 132.01 & 4 & 5 & 1 & 94.28 & 87.42 \\
\hline 5 & 10 & 0 & 22.53 & -22.78 & 5 & 5 & 1 & 43.13 & -40.72 \\
\hline 6 & 10 & 0 & 30.90 & -31.18 & 6 & 5 & 1 & 25.35 & -23.25 \\
\hline 1 & 12 & 0 & 11.85 & 12.07 & 7 & 5 & 1 & 17.53 & 16.50 \\
\hline 2 & 12 & 0 & 72.21 & 82.66 & 8 & 5 & 1 & 36.62 & 34.10 \\
\hline 3 & 12 & 0 & 58.45 & -67.44 & 1 & 7 & 1 & 101.65 & -104.44 \\
\hline 4 & 12 & 0 & 58.86 & -60.04 & 2 & 7 & 1 & 62.85 & 65.29 \\
\hline 5 & 12 & 0 & 14.44 & -19.23 & 3 & 7 & 1 & 52.22 & 58.44 \\
\hline 6 & 12 & 0 & 40.75 & 45.46 & 4 & 7 & 1 & 78.23 & -78.42 \\
\hline 1 & 14 & 0 & 38.25 & 46.45 & 5 & 7 & 1 & 93.63 & -89.82 \\
\hline 2 & 14 & 0 & 33.54 & -39.02 & 6 & 7 & 1 & 48.32 & 41.16 \\
\hline 1 & 16 & 0 & 0 & -5.30 & 7 & 7 & 1 & 17.53 & 18.69 \\
\hline 2 & 16 & 0 & 0 & -3.25 & 8 & 7 & 1 & 47.46 & -41.75 \\
\hline 3 & 16 & 0 & 83.14 & 90.53 & 1 & 9 & 1 & 68.28 & 74.48 \\
\hline 1 & 18 & 0 & 20.45 & 26.63 & 2 & 9 & 1 & 157.35 & -168.09 \\
\hline 2 & 18 & 0 & 12.40 & 15.85 & 3 & 9 & 1 & 71.31 & -69.71 \\
\hline 1 & 1 & 1 & 40.10 & -46.36 & 4 & 9 & 1 & 32.71 & 37.56 \\
\hline 2 & 1 & 1 & 175.95 & -177.03 & 5 & 9 & 1 & 46.16 & 46.33 \\
\hline 3 & 1 & 1 & 78.89 & 67.96 & 6 & 9 & 1 & 97.75 & -92.14 \\
\hline 4 & 1 & 1 & 18.42 & 14.18 & 7 & 9 & 1 & 41.38 & -42.89 \\
\hline 5 & 1 & 1 & 22.09 & -16.76 & 1 & 11 & 1 & 25.57 & -29.03 \\
\hline 6 & 1 & 1 & 93.21 & -81.74 & 2 & 11 & 1 & 46.38 & -57.93 \\
\hline 7 & 1 & 1 & 59.53 & 46.89 & 3 & 11 & 1 & 46.16 & 46.80 \\
\hline
\end{tabular}


Table 2. Continued

\begin{tabular}{r|r|r|r|r|r|r|r|r|r}
\hline$h$ & $k$ & $l$ & \multicolumn{1}{|c|}{$F_{o}$} & \multicolumn{1}{|c|}{$F_{c}$} & $h$ & $k$ & $l$ & \multicolumn{1}{|c|}{$F_{o}$} & \multicolumn{1}{c}{$F_{c}$} \\
\hline 4 & 11 & 1 & 0 & -1.26 & 3 & 4 & 2 & 84.65 & -82.56 \\
5 & 11 & 1 & 19.52 & -20.14 & 3 & 6 & 2 & 148.41 & 145.90 \\
6 & 11 & 1 & 28.82 & -32.17 & 3 & 8 & 2 & 42.28 & -41.25 \\
7 & 11 & 1 & 39.65 & 37.60 & 3 & 10 & 2 & 0 & -3.39 \\
1 & 13 & 1 & 59.81 & 67.02 & 3 & 12 & 2 & 15.49 & -16.85 \\
2 & 13 & 1 & 61.11 & 68.39 & 3 & 14 & 2 & 36.82 & -45.39 \\
3 & 13 & 1 & 40.10 & -47.92 & 3 & 16 & 2 & 62.17 & 67.64 \\
4 & 13 & 1 & 21.01 & -25.07 & 4 & 0 & 2 & 223.14 & 211.96 \\
5 & 13 & 1 & 52.44 & 57.46 & 4 & 2 & 2 & 35.56 & -23.64 \\
6 & 13 & 1 & 35.54 & 37.39 & 4 & 4 & 2 & 32.79 & -28.57 \\
1 & 15 & 1 & 39.01 & -47.25 & 4 & 6 & 2 & 20.35 & -17.48 \\
2 & 15 & 1 & 0 & -5.42 & 4 & 8 & 2 & 30.03 & 34.53 \\
3 & 15 & 1 & 25.78 & 32.40 & 4 & 10 & 2 & 116.40 & 124.27 \\
4 & 15 & 1 & 26.87 & 33.14 & 4 & 12 & 2 & 56.03 & -56.34 \\
5 & 15 & 1 & 38.13 & -42.44 & 5 & 2 & 2 & 79.36 & -73.96 \\
1 & 17 & 1 & 13.64 & -16.62 & 5 & 4 & 2 & 95.28 & 86.23 \\
2 & 17 & 1 & 10.17 & 2.49 & 5 & 6 & 2 & 50.47 & -44.39 \\
1 & 2 & 2 & 49.90 & -58.57 & 5 & 8 & 2 & 15.94 & 12.29 \\
1 & 4 & 2 & 137.44 & 134.43 & 5 & 10 & 2 & 15.05 & -17.50 \\
1 & 6 & 2 & 101.53 & -101.16 & 5 & 12 & 2 & 12.71 & 18.54 \\
1 & 8 & 2 & 17.32 & 13.28 & 5 & 14 & 2 & 12.91 & 16.53 \\
1 & 10 & 2 & 11.61 & -15.21 & 6 & 0 & 2 & 62.31 & -57.96 \\
1 & 12 & 2 & 23.75 & 31.33 & 6 & 2 & 2 & 54.40 & 45.96 \\
1 & 14 & 2 & 21.81 & 30.50 & 6 & 4 & 2 & 32.44 & -25.30 \\
1 & 16 & 2 & 71.78 & -71.49 & 6 & 6 & 2 & 13.93 & 5.84 \\
2 & 0 & 2 & 189.28 & -180.07 & 6 & 8 & 2 & 23.55 & 23.24 \\
2 & 2 & 2 & 112.12 & 110.08 & 6 & 10 & 2 & 54.07 & -52.68 \\
2 & 4 & 2 & 5.71 & 9.24 & 6 & 12 & 2 & 51.22 & 50.23 \\
2 & 6 & 2 & 49.58 & -56.14 & 7 & 2 & 2 & 38.72 & -30.62 \\
2 & 8 & 2 & 34.95 & 30.78 & 7 & 4 & 2 & 58.65 & -57.91 \\
2 & 10 & 2 & 21.67 & -23.40 & 7 & 6 & 2 & 99.23 & 84.68 \\
2 & 12 & 2 & 45.26 & 57.61 & 8 & 0 & 2 & 137.88 & 113.70 \\
2 & 14 & 2 & 50.67 & -61.27 & 8 & 2 & 2 & 41.67 & -23.06 \\
2 & 16 & 2 & 14.44 & 16.85 & 8 & 4 & 2 & 26.03 & -18.95 \\
3 & 2 & 2 & 69.97 & -62.26 & 8 & 6 & 2 & 11.69 & 13.78 \\
\hline & & & & & & & & & \\
\hline
\end{tabular}


Table 3. Atomic parameters for Ama2

\begin{tabular}{|c|c|c|c|c|}
\hline Atom & $x$ & $y$ & $z$ & $B$ \\
\hline $\mathrm{Sr}$ & 0.25 & $\begin{array}{r}0.2058 \\
\pm 0.0002\end{array}$ & $\begin{array}{l}0 * \\
0\end{array}$ & $\begin{array}{r}1.58 \\
\pm 0.08\end{array}$ \\
\hline V & 0.25 & $\begin{array}{r}0.3907 \\
\pm 0.0003\end{array}$ & $\begin{array}{r}0.5139 \\
\pm 0.0063\end{array}$ & $\begin{array}{r}1.74 \\
\pm 0.13 \\
\end{array}$ \\
\hline $\mathrm{Si}$ & $\begin{array}{r}0.0226 \\
\pm 0.0010\end{array}$ & $\begin{array}{r}0.1072 \\
\pm 0.0004\end{array}$ & $\begin{array}{r}0.4980 \\
\pm 0.0069\end{array}$ & $\begin{array}{r}1.59 \\
\pm 0.14 \\
\end{array}$ \\
\hline $\mathrm{O}_{1}$ & $\begin{array}{l}-0.0552 \\
\pm 0.0077\end{array}$ & $\begin{array}{r}0.1486 \\
\pm 0.0024\end{array}$ & $\begin{array}{r}0.2850 \\
\pm 0.0089\end{array}$ & $\begin{array}{r}3.39 \\
\pm 0.88\end{array}$ \\
\hline $\mathrm{O}_{2}$ & 0 & 0 & $\begin{array}{r}0.5363 \\
\pm 0.0095\end{array}$ & $\begin{array}{r}1.43 \\
\pm 0.43\end{array}$ \\
\hline $\mathrm{O}_{3}$ & 0.25 & $\begin{array}{r}0.0008 \\
\pm 0.0016\end{array}$ & $\begin{array}{r}-0.0308 \\
\pm 0.0088 \\
\end{array}$ & $\begin{array}{r}1.96 \\
\pm 0.51 \\
\end{array}$ \\
\hline $\mathrm{O}_{4}$ & 0.25 & $\begin{array}{r}0.1378 \\
\pm 0.0015 \\
\end{array}$ & $\begin{array}{r}0.4988 \\
+0.0245 \\
\end{array}$ & $\begin{array}{r}2.14 \\
\pm 0.48 \\
\end{array}$ \\
\hline $\mathrm{O}_{5}^{* *}$ & $\begin{array}{l}-0.0612 \\
\pm 0.0049\end{array}$ & $\begin{array}{r}0.1525 \\
\pm 0.0014\end{array}$ & $\begin{array}{r}0.7778 \\
\pm 0.0060\end{array}$ & $\begin{array}{r}0.16 \\
\pm 0.42 \\
\end{array}$ \\
\hline
\end{tabular}

* The $z$-coordinate of $\mathrm{Sr}$ was fixed throughout the refinements.

* Equivalent to $\mathrm{O}_{1}$ in the space group Amam.

actions of parameters occured between $x\left(\mathrm{O}_{1}\right)$ and $x\left(\mathrm{O}_{5}\right), y\left(\mathrm{O}_{1}\right)$ and $y\left(\mathrm{O}_{5}\right), z\left(\mathrm{O}_{1}\right)$ and $z\left(\mathrm{O}_{5}\right), B\left(\mathrm{O}_{1}\right)$ and $B\left(\mathrm{O}_{5}\right)$, and $k$ (scale factor) and $B(\mathrm{Sr})$. The correlation coefficients are respectively $-0.87,-0.82,0.74,-0.66$ and 0.78 . For the centrosymmetric case, these oxygens $\mathrm{O}_{1}$ and $\mathrm{O}_{5}$ are equivalent by a mirror operation. These interactions were even stronger if the above mentioned several reflections were not weighted zero.

The $R$ value for this refinement is lower than the Amam value by about one per cent. However, if the extra parameters introduced in the lower symmetry refinements are spurious, it would be plausible to expect $R$ value to be lower than the value for higher symmetry as discussed by CRUICKSHANK et al. (1962). In addition to this, as 
Table 4. Silicate dimensions for Amam and Ama2

\begin{tabular}{c|c|c}
\hline & $\begin{array}{c}\text { Amam } \\
\text { No. of } h k l=203 ; \\
\text { variables }=17 ; \\
R=0.086 .\end{array}$ & $\begin{array}{l}\text { Ama2 } \\
\text { No. of } h k l=190: \\
\text { variables }=26: \\
R=0.075 .\end{array}$ \\
\hline $\mathrm{Si}-\mathrm{O}_{1}$ & 1.60 & $1.40,1.74$ \\
$-\mathrm{O}_{2}$ & 1.58 & 1.59 \\
$-\mathrm{O}_{4}$ & 1.66 & 1.67 \\
$\mathrm{Si}-\mathrm{O}_{2}-\mathrm{Si}$ & $180^{\circ}$ & $165^{\circ}$ \\
$\mathrm{Si}-\mathrm{O}_{4}-\mathrm{Si}$ & $150^{\circ}$ & $149^{\circ}$ \\
$\mathrm{O}_{1}-\mathrm{Si}-\mathrm{O}_{1}$ & $115^{\circ}$ & $113^{\circ}$ \\
$\mathrm{O}_{1}-\mathrm{Si}-\mathrm{O}_{2}$ & $104^{\circ}$ & $103^{\circ}, 120^{\circ}$ \\
$\mathrm{O}_{2}-\mathrm{Si}-\mathrm{O}_{4}$ & $111^{\circ}$ & $110^{\circ}$ \\
$\mathrm{O}_{4}-\mathrm{Si}-\mathrm{O}_{1}$ & $112^{\circ}$ & $105^{\circ}, 103^{\circ}$ \\
\hline
\end{tabular}

shown in Table 4, where the silicate dimensions obtained by this refinement are compared with those for the centrosymmetric case, two lengths for chemically equivalent bonds are absurd. On these grounds, it will be reasonable to conclude that the space group of haradaite is Amam.

As observed in Table $1, \mathrm{Si}$ and $\mathrm{O}_{1}$ show somewhat higher temperature coefficients. They may be attributed to (a) thermal vibration of tetrahedra around the edge $\mathrm{O}_{2}-\mathrm{O}_{4}$ or (b) to positional disorders of $\mathrm{Si}$ and $\mathrm{O}_{1}$ which simulate the above mode of thermal vibration. For the latter case the space group $A m a 2$ may be assumed in a short range of the structure, the space group Amam being merely a result of averaging. If this is really the case, it will be possible to estimate the deviation of the bond angle of $\mathrm{O}_{2}$ by evaluating the mean square displacement $\overline{u^{2}}$ from the temperature coefficients. A calculation showed that the mean deviation amounts to $\pm 5^{\circ}$. However, as far as the Fourier projection $\rho(y z)$ is concerned no evidence is observed which supports the above mode of thermal vibration (Fig. 4). The higher temperature coefficients of $\mathrm{Si}$ and $\mathrm{O}_{1}$ may be resulted from errors in intensities. 


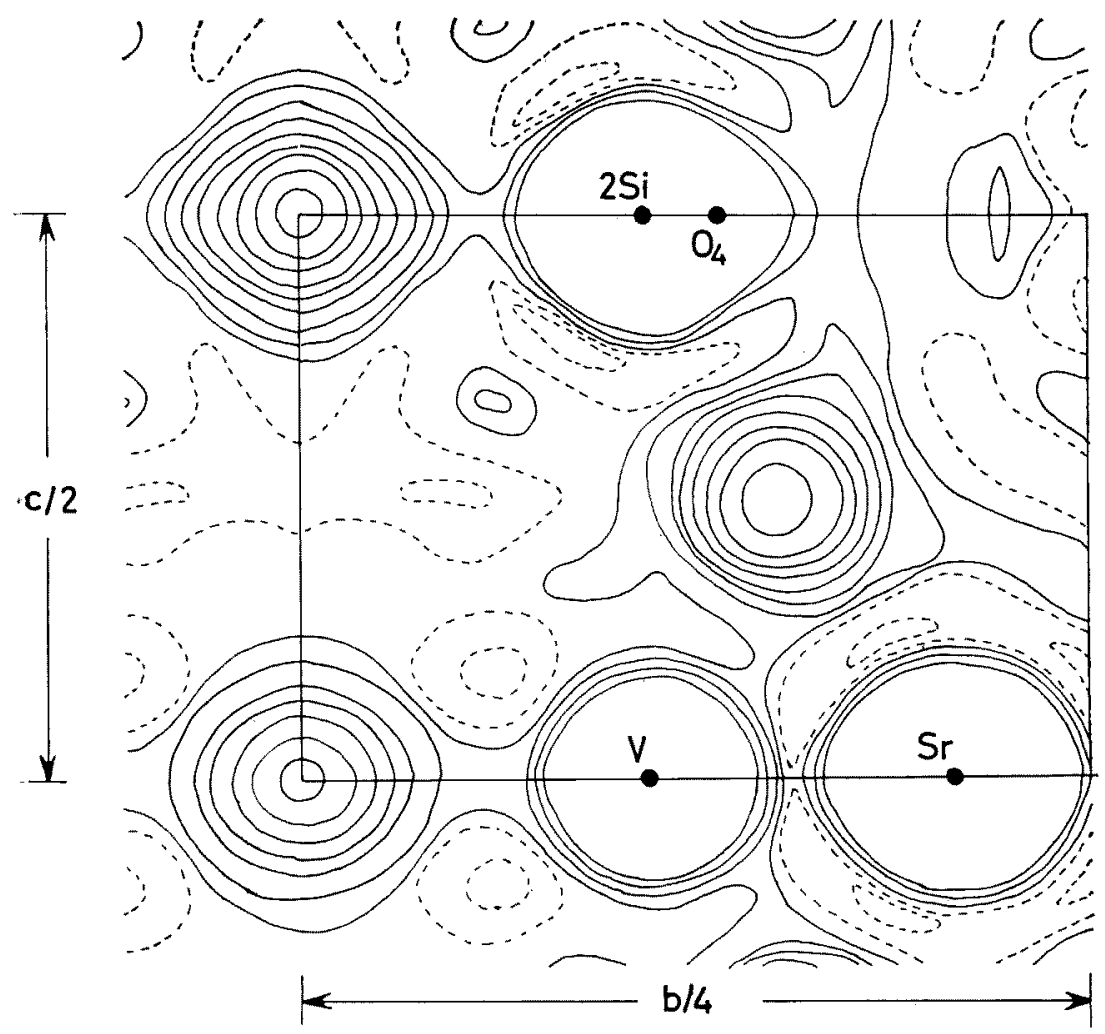

Fig. 4. Fourier projection along the $a$-axis. Contour intervals are one electron per $\AA^{2}$. Zero and negative contours are indicated by broken lines.

\section{Description of the structure}

The bond lengths, bond angles and interatomic distances are given in Table 5. In Fig. 5 and Fig. 6, the $c$-axis and $a$-axis projections of the structure of haradaite are given respectively. The salient feature of the structure is the $\mathrm{Si}_{4} \mathrm{O}_{12}$ chains running along the $a$-axis (Fig. 7). The configuration of the chain is similar to the component chains of the $\mathrm{Si}_{4} \mathrm{O}_{12}$ tubes found in narsarsukite (PYATENKo \& Pudovkina, 1960; PeAcor \& Buerger, 1962). Such a chain has been found in an isolated configuration in the structure of batisite 
Table 5. Bond lengths, bond angles and interatomic distances for haradaite

\begin{tabular}{c|l|l}
\hline $\mathrm{Si}-\mathrm{O}_{1}$ & $1.58_{8} \pm 0.03 \mathrm{~A}(2)^{*}$ & \\
$\mathrm{O}_{2}$ & $1.578 \pm 0.009$ & \\
$\mathrm{O}_{4}$ & $1.664 \pm 0.008$ & \\
\hline $\mathrm{V}-\mathrm{O}_{1}$ & $1.98_{6} \pm 0.03(4)$ & \\
$\mathrm{O}_{3}$ & $1.573 \pm 0.005$ & \\
\hline $\mathrm{Sr}-\mathrm{O}_{1}$ & $2.66_{9} \pm 0.03(4)$ & \\
$\mathrm{O}_{1}$ & $2.83_{3} \pm 0.03(4)$ & \\
$\mathrm{O}_{4}$ & $2.851 \pm 0.003(2)$ & \\
$\mathrm{O}_{3}$ & $3.016 \pm 0.003$ & mean : $2.79_{5}$ \\
\hline $\mathrm{O}_{1}-\mathrm{O}_{1}$ & $2.69_{4} \pm 0.04$ & \\
$\mathrm{O}_{1}-\mathrm{O}_{2}$ & $2.62_{3} \pm 0.03(2)$ & \\
$\mathrm{O}_{4}-\mathrm{O}_{2}$ & $2.667 \pm 0.002$ & Si-tetrahedra \\
$\mathrm{O}_{4}-\mathrm{O}_{1}$ & $2.57_{1} \pm 0.03$ & mean: $2.62_{5}$ \\
\hline $\mathrm{O}_{1}-\mathrm{O}_{3}$ & $2.90_{5} \pm 0.03(4)$ \\
$\mathrm{O}_{1}-\mathrm{O}_{1}(/ / c)$ & $2.63_{6} \pm 0.04(2)$ \\
$\mathrm{O}_{1}-\mathrm{O}_{1}(/ / a)$ & $2.70_{1} \pm 0.04(2)$ \\
\hline $\mathrm{O}_{1}-\mathrm{Si}-\mathrm{O}_{1}$ & $114.9 \pm 2.5^{\circ}$ & \\
$\mathrm{O}_{1}-\mathrm{Si}-\mathrm{O}_{2}$ & $104.1 \pm 1.5^{\circ}$ \\
$\mathrm{O}_{2}-\mathrm{Si}-\mathrm{O}_{4}$ & $110.7 \pm 0.8^{\circ}$ \\
$\mathrm{O}_{4}-\mathrm{Si}-\mathrm{O}_{1}$ & $111.7 \pm 1.5^{\circ}$ & \\
\hline $\mathrm{Si}-\mathrm{O}_{2}-\mathrm{Si}$ & $180^{\circ} * *$ \\
$\mathrm{Si}-\mathrm{O}_{4}-\mathrm{Si}$ & $150 \pm 0.9^{\circ}$ & \\
\hline
\end{tabular}

* Frequency of occurrence denoted in parentheses.

** Further discussion of the accuracy is given in the text.

(Nikitin \& BELOV, 1962).

In the structure of haradaite, these chains are joined together by vanadium to form a sheet which is closely related to that lamprophyllite. If $\mathrm{Ti}$ in the sheet of lamprophyllite is replaced by $\mathrm{V}$, and two sheets as such are combined so that the $\mathrm{Si}_{2} \mathrm{O}_{7}$ groupsmay form an infinite $\mathrm{Si}_{4} \mathrm{O}_{12}$ chain, the sheet of haradaite will be- 


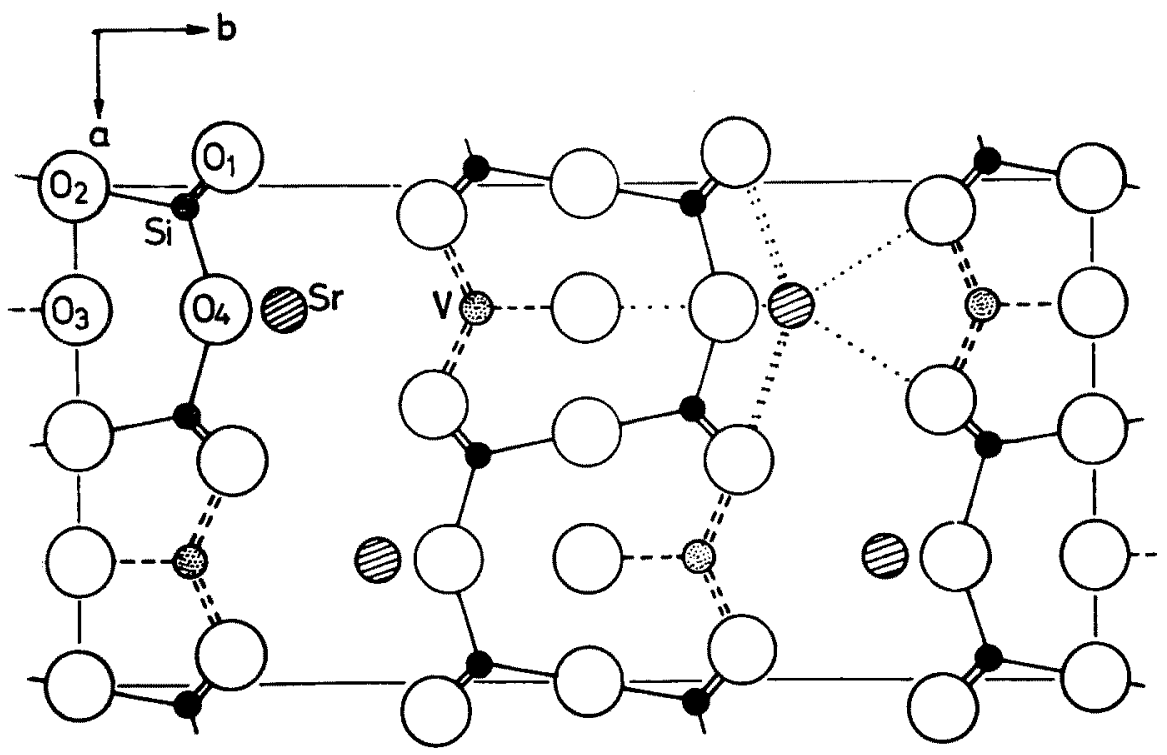

Fig. 5. The structure of haradaite projected along the $c$-axis.

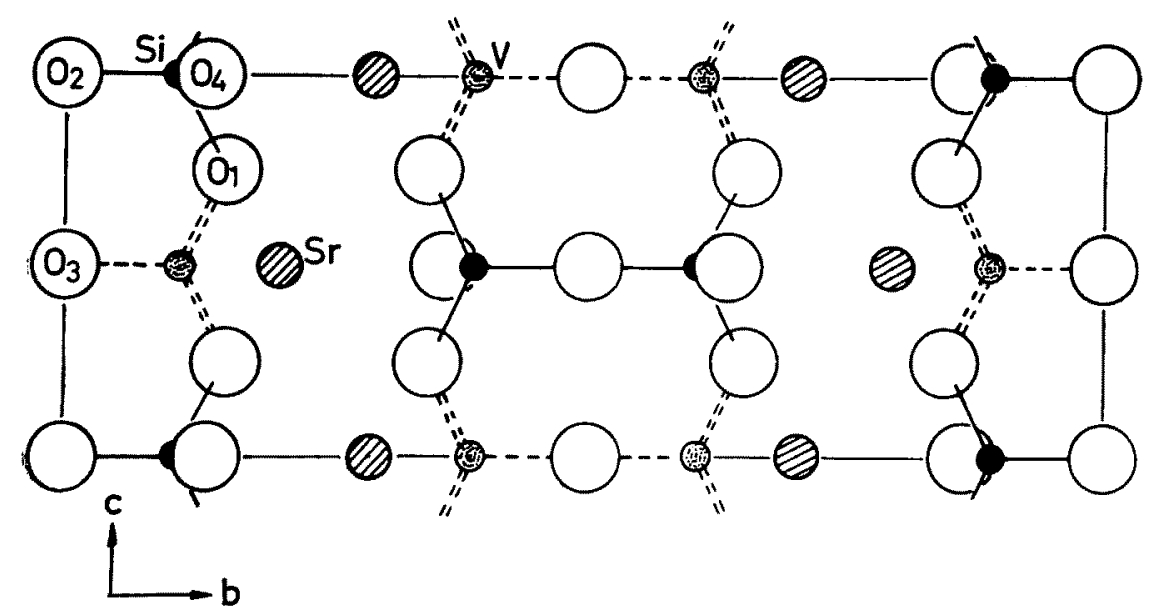

Fig. 6. The structure of haradaite projected along the $a$-axis.

The origin is shifted to $00 \frac{1}{2}$. 


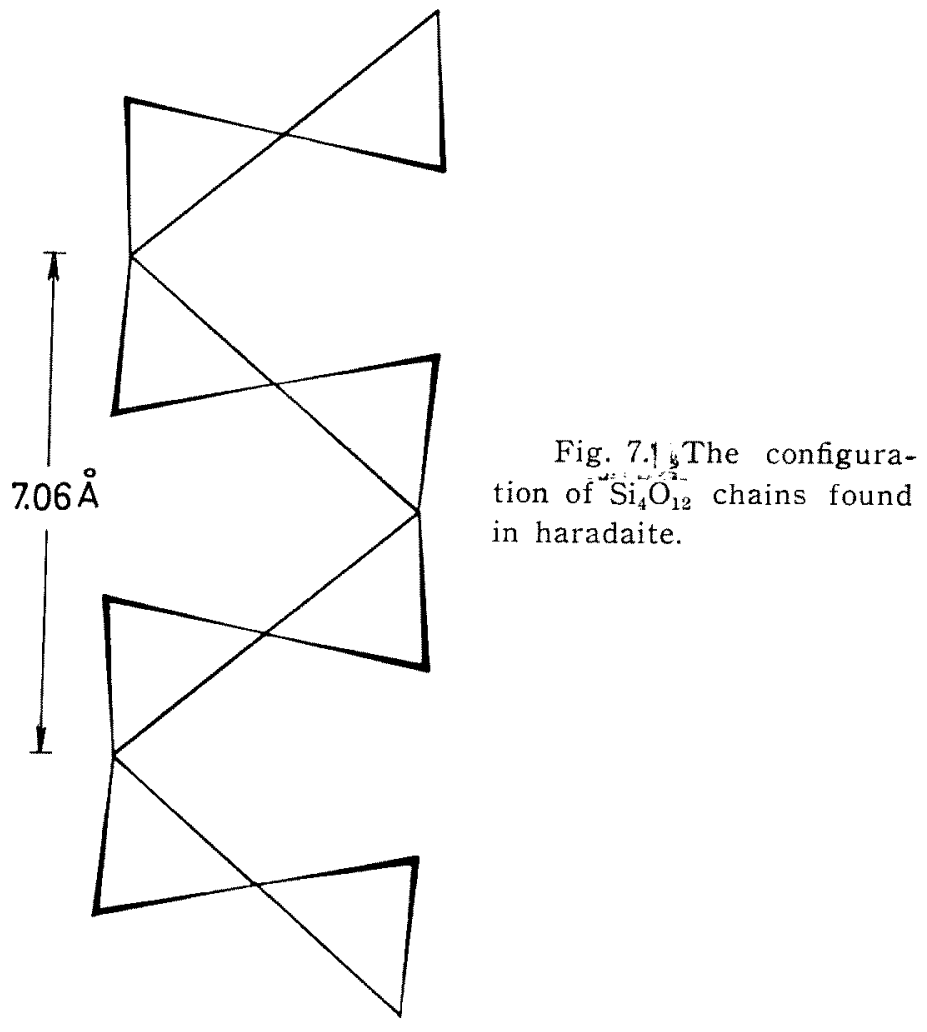

lamprophyllite

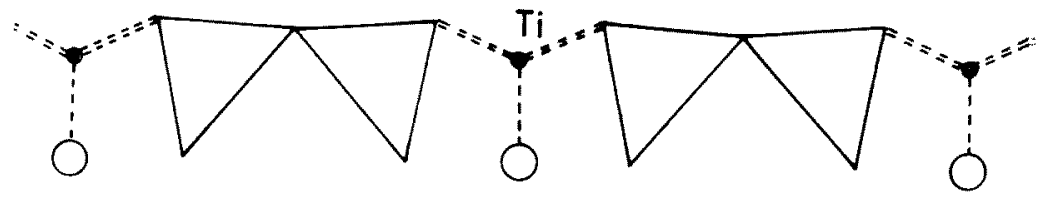

\section{haradaite}

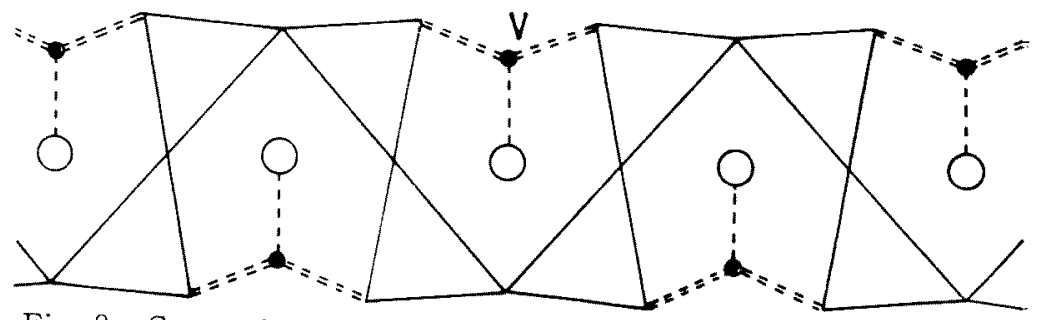

Fig. 8. Comparison of a cross section of lamprophyllite sheet and that of haradaite sheet. 
Table 6 . V-O bond lengths in some vanadium compounds

I. Six-fold coordination

Häggite, $\mathrm{H}_{6} \mathrm{~V}_{4} \mathrm{O}_{12}$ (Evans et al., 1960)

$$
\begin{array}{rl}
\mathrm{V}-\mathrm{O}_{1} & 1.82 \pm 0.02 \AA \\
\mathrm{O}_{2} & 1.97 \pm 0.02(2){ }^{*} \\
\mathrm{O}_{3} & 2.01 \pm 0.02(2) \\
\mathrm{O}_{3} & 2.06 \pm 0.02
\end{array}
$$

Doloresite, $\mathrm{H}_{8} \mathrm{~V}_{6} \mathrm{O}_{16}$ (EVANs et al., 1960)

$$
\begin{array}{rl}
\mathrm{V}_{1}-\mathrm{O}_{1} & 1.93(4) \\
\mathrm{O}_{2} & 1.98(2) \\
\mathrm{V}_{2}-\mathrm{O}_{2} & 1.70 \\
\mathrm{O}_{2} & 1.97(2) \\
\mathrm{O}_{4} & 2.01(2) \\
\mathrm{O}_{4} & 2.13
\end{array}
$$

II. Five-fold coordination

Vanadium pentoxide, $\mathrm{V}_{2} \mathrm{O}_{5}$ (BACHMANN et al., 1961)

$$
\begin{array}{cc}
\mathrm{V}-\mathrm{O}_{1} & 1.585 \pm 0.004 \\
\mathrm{O}_{3} & 1.780 \pm 0.002 \\
\mathrm{O}_{2}(2) & 1.878 \pm 0.002 \\
\mathrm{O}_{2}(3) & 1.878 \pm 0.002 \\
\mathrm{O}_{2}(1) & 2.021 \pm 0.003
\end{array}
$$

Vanadyl bisacetylacetonate, $\mathrm{VO}\left(\mathrm{C}_{5} \mathrm{H}_{7} \mathrm{O}_{2}\right)_{2}$ (DOdGE et al., 1961)

$\begin{array}{rr}\mathrm{V}-\mathrm{O}_{6} & 1.56 \pm 0.01 \\ \mathrm{O}_{2} & 1.97 \pm 0.01 \\ \mathrm{O}_{3} & 1.96 \pm 0.01 \\ \mathrm{O}_{4} & 1.98 \pm 0.01 \\ \mathrm{O}_{5} & 1.96 \pm 0.01\end{array}$

Potassium metavanadate monohydrate, $\mathrm{KVO}_{3} \cdot \mathrm{H}_{2} \mathrm{O}$ (CHRIsT et al., 1954)

$$
\begin{array}{cl}
\mathrm{V}-\mathrm{O}_{\mathrm{I}} & 1.63 \pm 0.02 \\
\mathrm{O}_{\mathrm{II}} & 1.67 \pm 0.02 \\
\mathrm{O}_{\mathrm{III}} & 1.93 \pm 0.02(2) \\
\mathrm{O}_{\mathrm{III}} & 1.99 \pm 0.02
\end{array}
$$

* Frequency of occurrence denoted in parentheses. Notation of atoms are those of original articles. 
derived (Fig. 8).

Vanadium atoms have a square pyramidal coordination slightly distorted. Among five bonds, the apical bond has a very short length suggesting that the bond is highly polarized. These bond lengths may be compared with those of vanadium compounds as given in Table 6, among which the vanadium atom in vanadyl bisacetylacetonate has a five-fold coordination similar to that in haradaite though in a different valence state. Another example of similar coordination has been reported for vanadium pentoxide (BACKMANN et al., 1961) although it is stated that oxygen coordination polyhedra in vanadium pentoxide is described as distorted trigonal bipyramidal rather than distorted tetragonal pyramidal. However, the distortion is so large that the coordination may also be described as a distorted square pyramid.

Sr atoms have eleven oxygen neighbors and are located between the sheets. Thus, the bulk of the structure explains well the perfect cleavage parallel to (010) and distinct ones parallel to (100) and (001). The existence of chain structure is consistent with the positive optical sign of this crystal.

\section{Quasi-layer silicates}

The sheet found in bafertisite consists of $\mathrm{Si}_{2} \mathrm{O}_{7}$ groups and $\mathrm{Ti}$ octahedra and its configuration is very similar to that in lamprophyllite. The structure of yoshimuraite, $(\mathrm{Ba}, \mathrm{Sr}, \mathrm{Na})_{2}(\mathrm{Mn}, \mathrm{Fe})_{2}(\mathrm{Ti}, \mathrm{Fe})$ $\mathrm{O}\left(\mathrm{Si}_{2} \mathrm{O}_{7}\right)(\mathrm{P}, \mathrm{S}) \mathrm{O}_{4}(\mathrm{OH})$ (WATANABE et al., 1961), probably has, with certainty, a sheet consisting of $\mathrm{Si}_{2} \mathrm{O}_{7}$ groups and $\mathrm{Ti}$ polyhedra though the structure is now under investigation. The structure of astrophyllite is another example which displays the sheet structure closely related to the lamprophyllite sheet. The lattice constants and chemical formulae of these minerals are summarized in Table 7 in which it can be observed that $\sim 5.4 \AA$ and $\sim 7.0 \AA$ are the characteristic repeats of these sheets. Though the lattice constants of astrophyllite are not 
Table 7. Lattice constants and chemical formulae of quasi-layer silicates

\section{Lattice constants}

$$
\begin{array}{ll}
\text { lamprophyllite* } & c=5.40, b=7.06, a=19.76, \beta=96.5^{\circ} \\
\text { bafertisite } & a=2 \times 5.30, b=2 \times 6.82, \quad c=12.47, \beta=119^{\circ} 30^{\prime} \\
\text { yoshimuraite } & c=5.39, \quad a=7.00, b=14.71, \quad \alpha=93.5^{\circ}, \beta=90.2^{\circ}, \gamma=95.3^{\circ} \\
\text { astrophyllite } & a=5.36, \quad b=11.76, \quad c=21.08, \quad \alpha=85.1^{\circ}, \beta=90.0^{\circ}, \quad \gamma=103.2^{\circ} \\
\text { haradaite } & c=5.33, \quad a=7.06, b=14.64
\end{array}
$$

\section{Chemical formulae}

\begin{tabular}{llll} 
& $\begin{array}{c}\text { Interlayer cation } \\
\text { and group }\end{array}$ & Octahedral sheet & $\begin{array}{c}\text { Quasi-silicate } \\
\text { sheet }\end{array}$ \\
lamprophyllite & $\mathrm{Ba}, \mathrm{Sr}, \mathrm{K}$ & $(\mathrm{Na}, \mathrm{Ti})(\mathrm{OH}, \mathrm{F})$ & $\mathrm{TiSi}_{2} \mathrm{O}_{8}$ \\
bafertisite & $\mathrm{Ba}$ & $\mathrm{Fe}_{2}(\mathrm{OH})_{2}$ & $\mathrm{TiSi}_{2} \mathrm{O}_{8}$ \\
yoshimuraite & $(\mathrm{Ba}, \mathrm{Sr}, \mathrm{Na})(\mathrm{P}, \mathrm{S}) \mathrm{O}_{4}$ & $\mathrm{Mn}_{2}(\mathrm{OH})$ & $\mathrm{TiSi}_{2} \mathrm{O}_{8}$ \\
astrophyllite & $\mathrm{K}_{1.5}$ & $(\mathrm{Mn}, \mathrm{Fe})_{3.5} \mathrm{O}_{1.5} \mathrm{H}_{x}$ & $\mathrm{Ti}_{2}\left(\mathrm{Si}_{2} \mathrm{O}_{7}\right)_{2}$ \\
haradaite & $\mathrm{Sr}, \mathrm{Ba}$ & & $\mathrm{VSi}_{2} \mathrm{O}_{7}$ \\
\hline
\end{tabular}

* An orthorhombic modification has been reported by TzE-CHung and Chien-Hung (1965).

characteristic of these sheets, the similarity between astrophyllite sheets and lamprophyllite sheets will be recognized in Fig. 9.

The structural schemes of these minerals are parallel with those of micas, especially biotites, in the respect that they have octahedral sheets which are sandwiched by the Si-Ti sheets as described above and, between these composite sheets, there exist such large cations as $\mathrm{Ba}$ or $\mathrm{K}$. Thus, the structure of these minerals may be considered as a derivative of the structure of biotites. In biotites, octahedral cations are mainly $\mathrm{Mg}, \mathrm{Fe}$ or $\mathrm{Li}$ and tetrahedral sites are occupied by $\mathrm{Si}$ and $\mathrm{Al}$ with the ratio, $3: 1$. The dimensions of the tetrahedral sheets are slightly larger than those of the octahedral sheets and the misfit between these is adjusted by rotations of tetrahedra. If tetrahedral sites are occupied solely by $\mathrm{Si}$, the tetrahedral sheet becomes slightly smaller than the octahedral one, causing a difficulty in adjusting the misfit (TAKEUCHI, 1965). The tetrahedral sheet for such a case, tends to corrugate, or takes a completely different configuration 
(a)

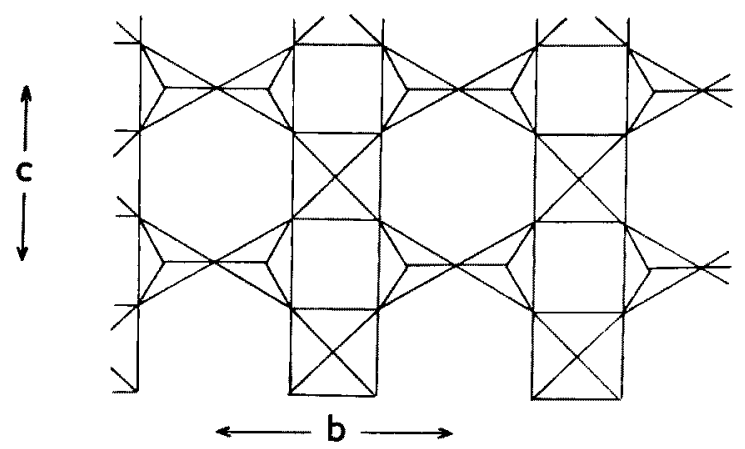

(b)

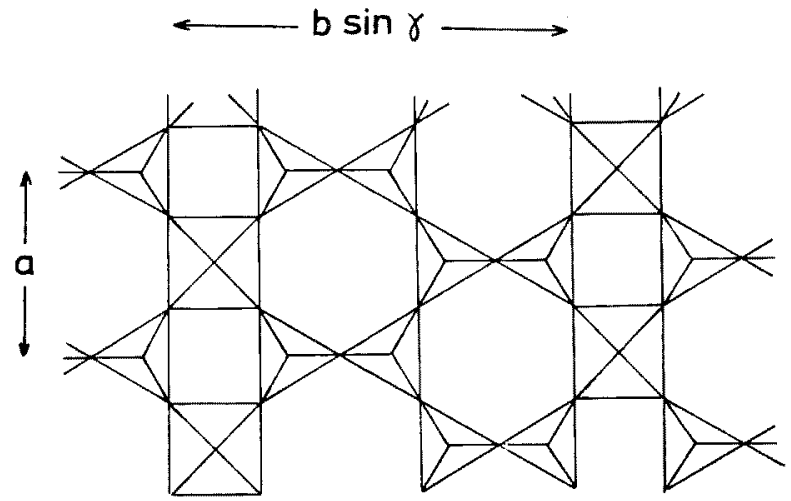

Fig. 9. The configurations of lamprophyllite sheet (a) and astrophyllite sheet (b).

as in pyrosmalite (TAKÉUCHI et al., 1963) or in the proposed structure for zeophyllite (CHALMERs et al., 1958), an extreme case where octahedral sites are occupied by $\mathrm{Ca}$. Another way of adjusting the misfit is to replace some of the tetrahedra by octahedra or some polyhedra of larger cations with high valency. This is exactly the case with the minerals listed in Table 7. Since the octahedral cations of these minerals are mainly $\mathrm{Na}, \mathrm{Ti}, \mathrm{Mn}$ or $\mathrm{Fe}$, the misfits between the $\mathrm{Si}_{4} \mathrm{O}_{10}$ 
tetrahedral sheet and the octahedral one are remarkable. By replacing some of the tetrahedra by $\mathrm{Ti}$ octahedra (or five-fold polyhedra), the misfit will be amended and the structure is retained to be of the mica type. These minerals are no longer classified as layer silicates as such because no continuous sheet of Si-tetrahedra is found there, but structurally and physically, they have layer structure closely relating to the mica structure. Thus these minerals may be called 'quasi-layer silicates'.

Although haradaite has no octahedral sheets, it can be classified into this group because it has $\mathrm{Si}-\mathrm{V}$ sheets of the lamprophyllite type. The relationship between haradaite and other quasi-layer silicates is somewhat similar to that between hexagonal $\mathrm{CaAl}_{2} \mathrm{Si}_{2} \mathrm{O}_{8}$ (TAKEUCHI \& Donnay, 1959) and micas. Rosenbuschite (SHIBAEVA et al., 1962) and seidozeite (SImonov et al., 1959) have sheets closely related to the lamprophyllite sheet. However the structures of these minerals consist of three-dimensional linkage of octahedra and, therefore, they do not fall into the category of quasi-layer silicates.

\section{A survey on Si-O bond lengths}

It is interesting to note that $\mathrm{Si}-\mathrm{O}$ bond lengths of haradaite show a considerable fluctuation (Table 5). Since the fluctuation seems to be significant, a survey on the Si-O bonds of other silicates was made separately for bridge bonds and for non-bridge bonds. The results are given in Table 8 where for each substance the mean of bridge bond lengths and that of non-bridge bond lengths are given. In the table, it will be observed that for the non-linear type, the bridge bonds are in the range between $1.63 \AA$ and $1.69 \AA$ giving the mean value, $1.65 \AA$, while non-bridge bonds are between $1.56 \AA$ and $1.63 \AA$, giving the mean value, $1.60 \AA$. If average is taken only for the structures of high accuracy like bustamite, rhodonite, wollastonite, orthopyroxene, narsarsukite and tourmaline, these values are respectively $1.641 \AA$ and $1.605 \AA$. The fact is in good accord with the $\pi$ - 
The structure of haradaite and a note on the Si-O

Table 8. Bond lengths of Si-O-Si bridge and Si-O non-bridge bonds in silicates

\begin{tabular}{|c|c|c|c|c|c|}
\hline \multicolumn{3}{|c|}{$\mathrm{Si}-\mathrm{O}-\mathrm{Si}$ angle $<180^{\circ}$} & \multicolumn{3}{|c|}{$\mathrm{Si}-\mathrm{O}-\mathrm{Si}$ angle $=180^{\circ}$} \\
\hline & $\begin{array}{l}\text { bridge } \\
\text { bond }\end{array}$ & $\begin{array}{c}\text { non- } \\
\text { bridge } \\
\text { bond }\end{array}$ & & $\begin{array}{l}\text { bridge } \\
\text { bond }\end{array}$ & $\begin{array}{l}\text { non. } \\
\text { bridge } \\
\text { bond }\end{array}$ \\
\hline $\begin{array}{l}\mathrm{Si}_{2} \mathrm{O}_{7} \\
\text { epidote }\end{array}$ & 1.65 & 1.63 & $\begin{array}{l}\text { thortveitite }{ }^{18} \\
\text { haradaite** }\end{array}$ & $\begin{array}{l}1.61 \\
1.58\end{array}$ & $\begin{array}{l}1.63 \\
1.60\end{array}$ \\
\hline tilleyite 2 & 1.65 & 1.60 & low-cordierite ${ }^{12 *}$ & 1.593 & 1.630 \\
\hline zoisite & 1.65 & 1.62 & & & \\
\hline $\begin{array}{l}\mathrm{SiO}_{3} \\
\text { bustamite }\end{array}$ & 1.642 & 1.604 & & & \\
\hline clinoenstatite $^{6}$ & 1.69 & 1.61 & & & \\
\hline orthopyroxene ${ }^{14}$ & 1.636 & 1.606 & & & \\
\hline rhodonite ${ }^{6}$ & 1.648 & 1.609 & & & \\
\hline pigeonite $^{5}$ & 1.65 & 1.59 & & & \\
\hline wollastonite ${ }^{7}$ & 1.655 & 1.598 & & & \\
\hline $\begin{array}{l}\mathrm{Si}_{4} \mathrm{O}_{11} \\
\text { actinolite }^{9}\end{array}$ & 1.67 & 1.61 & & & \\
\hline crocidolite $^{10}$ & $\begin{array}{l}1.65 \\
\sim 1.66\end{array}$ & $\begin{array}{l}1.59 \\
\sim 1.60\end{array}$ & & & \\
\hline $\begin{array}{l}\mathrm{Si}_{4} \mathrm{O}_{12} \\
\text { narsarsukite }\end{array}$ & 1.626 & 1.601 & & & \\
\hline $\begin{array}{l}\mathrm{Si}_{6} \mathrm{O}_{18} \\
\text { tourmaline }\end{array}$ & 1.637 & 1.605 & & & \\
\hline $\begin{array}{l}\mathrm{Si}_{4} \mathrm{O}_{10} \\
\text { polylithionite }\end{array}$ & 1.64 & 1.56 & & & \\
\hline
\end{tabular}

1. Itro et al., 1954; 2. Smith, 1953; 3. Fesenko et al., 1956; 4. Peacor \& Buerger, 1962b; 5. Morimoto et al., 1960; 6. Peacor \& NiIzeki, 1963; 7. Peacor \& Prewitt, 1963; 8. Buerger et al., 1962; 9. Zussman, 1955; 10. Whittaker, 1949; 11. TAKEDA, 1965; 12. GibBs, 1966; 13. Cruickshank et al., 1962; 14. Ghose, 1965; 15. Peacor \& Buerger, 1962a.

* Al-rich tetrahedra are excluded.

** Both linear and non-linear bonds are involved in a tetrahedron of haradaite. The bond length of the non-linear bond is not listed in this table.

bonding theory for $\mathrm{XO}_{4}$ ions developed by CRUICKSHANK (1961). On the contrary, for the linear Si-O-Si bonding type, though only three examples are available, bridge bonds tend to be shorter than non- 
bridge ones. BARCLAY and COX (1960) reported that the bridge bonds and non-bridge bonds of the $\mathrm{Si}_{2} \mathrm{O}_{7}$ groups in hemimorphite are respectively $1.72 \AA$ and $1.61 \AA$. Further refinement by CRUICKSHANK (1965), however, has shown that they are respectively $1.626 \AA$ and $1.623 \AA$, the difference being negligible. This is explained by the fact that the structure of hemimorphite is actually a three-dimensional framework of $\mathrm{Zn}$ - and $\mathrm{Si}$ - tetrahedra. It should therefore be noted that for silicate structures except orthosilicates and those with threedimensional frameworks, the over-all mean of tetrahedral bond lengths is less meaningful.

Some exceptions are observed especially in clay minerals. The data on kaolinite which are based on a two-dimensional refinement are $1.62_{1} \pm 0.02 \AA$ for bridge bonds and $1.63_{2} \pm 0.02 \AA$ for non-bridge bonds (DRITS \& KASHAEV, 1960). While, those of dickite, a polymorphous form of kaolinite, are $1.62_{3} \pm 0.017 \AA$ for bridge bonds and $1.61_{5} \pm 0.017 \AA$ for non-bridge ones (NEWNHAM, 1961). For these cases, the differences between two bond types are not significant. The fact may be attributed to the existence of some particular interactions. between $\mathrm{OH}$ layers and basal oxygen (bridge oxygen) layers. As a matter of fact, other clay minerals in which $\mathrm{Si}$ is replaced by $\mathrm{Al}$ for some extent also have the similar tendency of the bond lengths, namely non-bridge bonds are equal to the bridge ones or tend to belonger than bridge ones, though most of the data are based on twodimensional refinements.

We wish to express our gratitude to Professor R. Sadanaga for critical discussions and reading the manuscript. We are indebted to Professor Takeo Watanabe, Department of Geology, University of Tokyo, for furnishing materials and necessary information, and to Dr. J. Ito for discussions. Our thanks are also due to Dr. Y. Iitaka Pharmaceutical Department, University of Tokyo, and Dr. M. Ohmasa, for their help in computations. and to Dr. S. Hosoya and Dr. T. Imura, Institute for Solid State Physics, University of Tokyo, where. 
experiments were in part performed.

\section{REFERENCES}

Bachmann, H. G., Ahmed, F. R. \& Barues, W. H. (1961). Z. Kristallogr., 115, $110-131$.

Barclay, G. A. \& Cox, E. G. (1960). Z. Kristallogr., 113, 23-29.

Buerger, M. J., Burnham, C. W. \& Peacor, D. R. (1962). Acta Cryst., 15 , 583-590.

Chalmers, R. A., Dent, L.S. \& Taylor, H. F. W. (1958). Min. Mag., 31, 726735.

Christ, C. L., Clark, J. R. \& Evans, H. T. Jr. (1954). Acta Cryst., 7, 801-807. Cruickshank, D. W. J. (1961). J. Chem. Soc., 5486-5504.

Cruickshank, D. W. J., Lynton, H. \& Barclay, G. A. (1962). Acta Cryst., 15, 491-498.

CRUICKSHANK, D. W. J. (1965), private communication.

Dodge, R. P., Templeton, D. H. \& Zalkiv, A. (1961). J. Chem. Phys., 35, 5567.

Drits, V. A. \& Kashaev, A. A. (1960). Kristallografia, 5, 224-227.

Evans, H. T. Jr. \& Mrose, Mary E. (1960). Amer. Min., 45, 1144-1160.

Fesenko, E. G., Rumanova, I. M. \& Belov, N. V. (1956). Kristallografia, 1, $171-196$.

Gibis, G. V. (1966). Amer. Min., 51, 1068-1087.

Ghose, S. (1965). Z. Kristallogr., 122, 81-99.

Hosoya, S. \& Satake, S. (1965). Tech. Rep. Inst. Solid State Phys., Ser. B,

No. 8, 1-10, Univ. Tokyo.

ITo, J. (1965). Min. Jour., 4, 299-316.

Ito, T., Morimoto, N. \& Sadanaga, R. (1954). Acta Cryst., 7, 53-59.

Morimoto, N., Appleman, D. E. \& Evans, H. T. Jr. (1960). Z. Kristallogr., $114,120-147$.

Newnham, R. E. (1961). Min. Mag., 32, 683-704.

Nikitin, A. V. \& Belov, N. V. (1962). Dok. Akad. Nauk SSSR, 146, 1401-1403.

Peacor, D. R. \& Buerger, M. J. (1962a). Amer. Min., 47, 539-556.

Peacor, D. R. \& Buerger, M. J. (1962b). Z. Kristallogr., 117, 331-343.

PeAcor, D. R. \& Nilzeki, N. (1963). Z. Kristallogr., 119, 98-116.

Peacor, D. R. \& Prewitt, C. T. (1963). Amer. Min., 48, 588-596.

Pyatenko, Y.A. \& Pudovisina, Z. V. (1960). Kristallografia, 5, 673-573.

Shibaeva, R. P., Simonov, V.I. \& Belov, N. V. (1963). Kristallografia, 8, $506-516$.

Simonov, V. I. \& Belov, N. V. (1959). Kristallografia, 4, 163-175.

Sмith, J.V. (1953). Acta Cryst., 6, 9-18.

TAKEDA, H. (1965). private communication.

Takeuchi, Y. \& Donnay, Gabrielle (1959). Acta Cryst., 12, 465-470. 
Takeuchi, Y., Kawada, I. \& Sadanaga, R. (1963). Acta Cryst., 16, A 16.

TAKEUChI, Y., Ghose, S. \& NowACKI, W. (1965). Z. Kristallogr., 121, 321-348. TAKEUCH, Y. (1965). Clays and Clay Minerals, 13th Conf. (1964), pp. 1-25, Pergamon Press, New York.

Tze-Chung, P. \& Chien-Hung, C. (1965). Scientia Sinica, 14, 1827-1840. Watanabe, T., Takeuchi, Y. \& ITo, J. (1960). Min. Jour., 3, 156-167.

WhitTaker, E. J. W. (1949). Acta Cryst., 2, 312-317.

WOODROW, P. J. (1963). Acta Cryst., 16, A16-17.

WOODROW, P. J. (1964). Nature, 204, 375.

Ya-Hsien, K. Simonov, V.I. \& Belov, N. V. (1963). Dok. Akad. Nauk SSSR, 149, 1416-1419.

Zussman, J. (1955). Acta Cryst., 8, 301-308.

Received 22 October 1966. 